EPJ manuscript No.

(will be inserted by the editor)

\title{
GCM Analysis of the Collective Properties of Lead Isotopes with Exact Projection on Particle Numbers
}

\author{
P.-H. Heenen ${ }^{1}$, A. Valor ${ }^{1}$, M. Bender ${ }^{1}$, P. Bonche ${ }^{2}$, and H. Flocard ${ }^{3}$ \\ 1 Service de Physique Nucléaire Théorique, Université Libre de Bruxelles CP229, B-1050 Bruxelles \\ 2 Service de Physique Théorique, CE Saclay, F-91191, Giv-sur-Yvette Cedex \\ 3 Institut de Physique Nucléaire, F-91406 Orsay Cedex
}

June 12001

\begin{abstract}
We present a microscopic analysis of the collective behaviour of the lead isotopes in the vicinity of ${ }^{208} \mathrm{~Pb}$. In this study, we rely on a coherent approach based on the Generator Coordinate Method including exact projection on $N$ and $Z$ numbers within a collective space generated by means of the constrained Hartree-Fock BCS method. With the same Hamiltonian used in HF+BCS calculations, we have performed a comprehensive study including monopole, quadrupole and octupole excitations as well as pairing vibrations. We find that, for the considered nuclei, the collective modes which modify the most the conclusions drawn from the mean-field theory are the octupole and pairing vibrations.
\end{abstract}

PACS.

\section{Introduction}

Mean-field methods have been extensively useed to study a wide range of nuclear properties. There exist now several variants which although relying on different physical approaches, lead to rather similar results. Among the methods most extensively used in the last years, one can men' tion those based on Skyrme (zero-range) [1,2] interactions, or on the Gogny force (finite-range interaction) [3, 1 , 5 , the density-functional approach [6] and the relativistic meanfield method based on an effective Lagrangian [7,8].

One of the key ingredient in all these methods is the way the nucleon-nucleon interaction is treated. Either a density- functional or a relativistic Lagrangian is globally adjusted to some classes of nuclei and/or nuclear matter properties. Henceforth, it should be used without readjustment for any specific problem relevant to this class of properties. Mean-field methods attempt to reproduce nuclear many body properties within a description which takes into account the one body density only. Expectedly, the data accessible to these studies is mostly limited to that corresponding to one body operators, such as the shape deformations or the radii. To that already large set one can add the total binding energy for which the Hartree-Fock (HF) or Hartree-Fock Bogoliubov (HFB) minimization principle is explicitly designed. In practice, other observables are sometimes also included in the fit of the functionals or lagrangians. A typical example is the effective nucleon mass which is related to the density of single particle levels at the Fermi surface. To obtain a density similar to that deduced from the results of pickup and stripping reactions, the effective mass of the interaction should be close to the free nucleon mass. On the other hand, it has been shown that RPA correlations which are the simplest type of correlations beyond $\mathrm{HF}$, increase the value of the effective mass [9].. To account for such facts, two approaches have been utilized in non-relativistic studies as soon as the nuclear energy density approach has been shown to be flexible enough to reproduce nuclear properties with a good accuracy. In one of them, one tries to define the functional so as to avoid a double counting of the correlations. This implies that the choice of the effective parameters leaves room for the improvement expected from a further inclusion of RPA correlations. For instance, the mean-field value of the effective nucleon mass is adjusted to a value smaller than the bare mass so that RPA correlations may enhance it to the experimental value. In the past, this has been the most standard approach to define effective interactions used in the calculation of the energy functional. Another strategy consists in the construction of functionals which are in a sense "doubly" effective since they are expected to take into account the effect of all correlations beyond meanfield on a specified set of nuclear properties. Then, for such properties, the mean-field calculation should yield results directly comparable to data. Two successful examples of the latter strategy are the interaction of Tondeur et al. [10 which reproduces most known nuclear masses with high accuracy and that of Brown [11] which yields single particle level schemes to be used in subsequent shell model calculations.

In this work, we consider a functional belonging to the the first family in order to investigate how collective 
modes modify the mean-field properties. For this analysis, we rely on the generator coordinate method (GCM) taking into account several collective variables. In the past, such an approach has been shown to be suited for a description of low lying collective states related to the quadrupole 12 or the octupole [13] degree of freedom. A study of the coupling of the dipole and the octupole modes in ${ }^{152} \mathrm{Sm}$ and in the superdeformed band of ${ }^{190} \mathrm{Hg}$ [14 has also demonstrated the ability of the GCM to describe in a single calculation the properties of the ground state, of the low lying collective states and of the giant dipole resonance. This method seems therefore to be an appropriate tool to study the effect of correlations beyond a mean-field approach on the ground state properties of a nucleus.

As test case, we have selected five $\mathrm{Pb}$ isotopes around the magic number $N=126$. These nuclei are in principle well described by mean-field theories. The evolution of the two neutron separation energy at a magic number is representative of the shell gap. We will therefore determine how much it is affected by different types of collective correlations. Our aim is to be as systematic as possible within the GCM and to investigate the effect of all collective nuclear shape degrees of freedom which are believed to be important for these nuclei : the monopole, quadrupole and octupole modes.

Moreover, our study also includes the pairing vibration mode. Indeed, as part of this work, we analyze the validity of a commonly used treatment of pairing correlations beyond the BCS theory. Since BCS correlations in ${ }^{208} \mathrm{~Pb}$ vanish, one often attempts to describe them by means of an approximate variation after projection on the nucleon numbers. In the following, we test the validity of the Lipkin Nogami (LN) prescription [15,16,17 by performing an exact projection of the BCS wave functions. A further study of pairing vibrations also naturally provides an alternative approximate variation after projection and allows to test the quality of the LN prescription.

The organization of this work is as follows. In Sect.2, we present the dynamical ingredients (Hamiltonian and collective spaces) and recall the method while introducing our notations. The Sect. 3 discusses our results. In the last section, we summarize the major conclusions and indicate possible extensions of the present work and promising outlooks.

\section{Method}

In this section, we briefly review the now well established non-relativistic microscopic framework which leads to the results on lead isotopes presented in Sect. 3. It provides a natural setup for a treatment of i) the mean-field properties by means of standard HF + BCS or HFB methods with effective Hamiltonians and ii) the collective dynamics of any amplitude by means of the GCM.

\subsection{The Effective Hamiltonian and the Mean-Field Equations}

Over the last quarter of century, it has been acknowledged that the separation of the nuclear binding energy at the mean-field level in Hartree-Fock and pairing components in the spirit of the energy density approach provides a fruitful starting point for an investigation of the static and collective properties of nuclei. Without requiring completely consistency, since the Hartree-Fock and pairing fields remain coupled through the one-body density matrix, this approach deviates from the strict HFB theory as it can be performed with the Gogny [3] or SkP [18] interactions. Still, in nuclear physics, the energy density approach is often formulated in a way which preserves contact with the notion of effective force. Then, as it will be the case here, two interactions are used; one in the particle-hole (Hartree-Fock) channel and one in the particle-particle (pairing) channel. The introduction of these two effective forces yields additional flexibility in the phenomenological description of nuclear properties.

Historically, the structure of the particle-hole interaction, which in heavy nuclei accounts for more than $99 \%$ of the binding energy, has been investigated first. In the present work, we use one of the latest versions of the well established line of Skyrme interactions: the SLy4 parametrization [19] designed to describe properties of nuclei (light and heavy), near the stability line or in the neutron rich region up to the drip line with a similar accuracy. Here, we are concerned with stable and long lived lead isotopes. The quality of SLy4 for such non exotic nuclei has been tested on numerous examples 19,20,2,21. The force which acts in the pairing channel and defines the corresponding terms in the energy density is taken from Refs. [22,20]. It is a zero-range interaction with a dependence on the nucleon space density. This dependence ensures that the particle-particle two-body matrix elements are sensitive to the behavior of the single-particle orbitals mostly located at the surface of the nucleus. This interaction has been tested on physical issues sensitive to pairing properties, such as the evolution of moment of inertia in extended rotational bands as have been observed for instance in superdeformed 22] and superheavy 23 nuclei.

Along a series of isotopes or isotones, as either $N$ or $Z$ approaches a magic value, the mean-field method predicts a sudden collapse of pairing. It is now established that the magnitude of this transition overemphasizes the lowering of pairing correlations which must be expected when the single particle density at the Fermi surface decreases significantly. Indeed, even in magic nuclei when the mean value of the order parameter vanishes, it is expected that fluctuations of this order parameter (in phase and magnitude) should still influence nuclear properties. The importance of this so-called dynamical pairing (as opposed to static pairing in situations when the HFB and HF solutions differ from each other) is one of the questions that we investigate below within the GCM formalism. Lipkin and Nogami have proposed a prescription which attempts to correct the mean-field for the effects of the phase fluctuations of the order parameter [15, 16, 17. This still not well 


\begin{tabular}{lll}
\hline Vibration & Operator \\
\hline Monopole & $Q_{00} \propto \sum_{i} r_{i}^{2}$ & \\
Quadrupole & $Q_{2 m} \propto \sum_{i} r_{i}^{2} Y_{2 m}\left(\theta_{i}, \varphi_{i}\right)$ & $, m=0, \pm 2$ \\
Octupole & $Q_{30} \propto \sum_{i} r_{i}^{2} Y_{30}\left(\theta_{i}, \varphi_{i}\right)$ & \\
\hline
\end{tabular}

Table 1. Constraining operators used to construct the collective spaces associated with the lowest isoscalar nuclear shape vibrations. The quantities $r_{i}, \theta_{i}$ and $\varphi_{i}$ refer to the spherical coordinates of the nucleon $i$ in an intrinsic reference frame whose origin is at the nucleus center of mass. The $Y_{l m}$ are the standard spherical harmonics.

understood prescription has nevertheless been shown to lead to global improvement (over the mean-field theory) for the description of nuclear properties related to pairing. We will therefore also present results obtained with the Lipkin Nogami prescription which is also sometimes presented as an approximation to the variation after projection method (VAP).

To conclude this section let us mention a technical point: when pairing correlations are present, whether at the static level (HFB) or because the Lipkin Nogami prescription has been implemented, we have taken care that no orbital of the continuum is occupied in the HFB wave function. In practice, this is achieved by means of a cutoff which excludes all orbitals whose single particle energy is $5 \mathrm{MeV}$ above the Fermi energy, from the active pairing space.

\subsection{The Construction of the Collective Spaces}

In this work, the set of collective wave functions $\{|\Phi(q)\rangle\}$ are determined by self consistent methods ( $q$ denotes the value of the collective variable of interest). For the modes associated with isoscalar shape vibrations (monopole, quadrupole, octupole), these spaces are obtained by constrained $\mathrm{HF}+\mathrm{BCS}$ calculations with the appropriate one-body operator as listed in Table 1 and $q$ is the expectation value of the multipole moment operator $q=\left\langle\Phi(q)\left|Q_{j m}\right| \Phi(q)\right\rangle$

For the vibrations in the pairing space the collective coordinate must be related to some global (complex) pairing gap. Since, as is discussed in Sect.2.3, the fluctuations associated with the phase of the gap are taken into account by an exact projection after the $\mathrm{HF}+\mathrm{BCS}$ variation, there only remains to describe the fluctuations of the gap magnitude. To build the associated collective space, there is no unique prescription For instance, one may consider generating the space by means of HFB calculations with a constraint on the fluctuations of the neutron and proton numbers (i.e. the operators $\Delta \hat{N}_{\mu}^{2}, \mu=n, p$ ) [24].

In order to escape the technical difficulties associated with the two-body nature of this operator, we have adopted a second method. We construct the space by means of non constrained $\mathrm{HF}+\mathrm{BCS}$ calculations using the same functional (with the SLy4 parametrization) for the mean-field part and replacing the pairing functional by that associated with an auxiliary Hamiltonian depending on two real gaps parameters $\Delta_{n}$ and $\Delta_{p}$ :

$\hat{H}_{P}\left(\Delta_{n}, \Delta_{p}\right)=\frac{\Delta_{n}}{2} \sum_{\mu=1}^{N} a_{n \mu}^{\dagger} a_{n \bar{\mu}}^{\dagger}+\frac{\Delta_{p}}{2} \sum_{\mu=1}^{Z} a_{p \mu}^{\dagger} a_{p \bar{\mu}}^{\dagger}+$ c.c.,

where $a_{\tau \mu}^{\dagger}$ is the creation operator of the nucleon of isospin $\tau$ in the individual state $\mu$ and where $\bar{\mu}$ denotes the time reversed orbital. The variation of the corresponding total functionals generates a set of BCS states $\left\{\left|\Phi\left(\Delta_{n}, \Delta_{p}\right)\right\rangle\right\}$. Once this collective space has been determined, all further calculations, (expectation values and GCM matrix element calculations (see Sect. 2.3)) are performed with the Hamiltonian described in Sect. 2.1. This method has already been used in Ref. 25] to investigate the influence of dynamical pairing on tunneling probabilities between the superdeformed and normal wells in ${ }^{192} \mathrm{Hg}$.

The GCM method involves a non orthonormal collective basis. The two prescriptions for constructing the pairing collective space sketched above give should therefore lead to similar results (collective energy and wave functions) once the bases are large enough. In addition, the GCM method does not explicitly depend on which collective coordinates have been selected to span the total collective space. As long as the mapping is one to one, rather than by $\left(\Delta_{n}, \Delta_{p}\right)$, we may as well choose to plot the GCM collective properties in terms of the coordinates $\left(\left\langle\Delta \hat{N}_{n}^{2}\right\rangle,\left\langle\Delta \hat{N}_{p}^{2}\right\rangle\right)$ where $\langle\hat{A}\rangle$ denotes the expectation value of the operator $\hat{A}$ in the state $\left|\Phi\left(\Delta_{n}, \Delta_{p}\right)\right\rangle$ (or by any other set of coordinates).

\subsection{The Projection and the Collective Dynamics}

The Lipkin-Nogami prescription determines a BCS wave function which, in priciple, takes into account the pairing fluctations associated with the phase $\phi$ of the pairing. This wave function can be used directly to evaluate the expectation value of any operator as proposed in the original articles of Lipkin and Nogami. In this work, such values will be refered to as L.N. One can also consider that this BCS state is an approximation of the exact VAP state. In order to test the validity of this assumption, one must then extract from the LN-BCS state its component with correct (i.e. $N_{0}$ ) particle number by means of a projection $\hat{P}_{N_{0}}$. Henceforth, the results associated with such a two-step approach will be labeled L.N. (proj.)

The projection

$$
\hat{P}_{N_{0}}=\frac{1}{\pi} \int_{-\frac{\pi}{2}}^{\frac{\pi}{2}} \mathrm{~d} \phi \mathrm{e}^{i \phi\left(\hat{N}-N_{0}\right)}
$$

involves an integral over $\phi$ with the weight factor $\mathrm{e}^{-i \phi N_{0}}$. From the mean-field wave functions $\{|\Phi(q)\rangle\}$ associated with the value $q$ of the collective variable, we build the states

$$
\left|\Phi_{N_{0}}(q)\right\rangle=\hat{P}_{N_{0}}|\Phi(q)\rangle
$$


which form the non orthogonal projected collective basis $\left\{\left|\Phi_{N_{0}}(q)\right\rangle\right\}$ The expectation value $\mathcal{E}(q)$ of the Hamiltonian in this basis is the projected deformation energy curve:

$$
\mathcal{E}(q)=\frac{\left\langle\Phi_{N_{0}}(q)|\hat{H}| \Phi_{N_{0}}(q)\right\rangle}{\left\langle\Phi_{N_{0}}(q) \mid \Phi_{N_{0}}(q)\right\rangle}=\frac{\left\langle\Phi(q)\left|\hat{H} \hat{P}_{N_{0}}\right| \Phi(q)\right\rangle}{\left\langle\Phi(q)\left|\hat{P}_{N_{0}}\right| \Phi(q)\right\rangle} .
$$

Several such curves are discussed in Sect 3 . The value at the minima of $\mathcal{E}(q)$ yields an energy corresponding to a restricted VAP (a variation limited to the subspace spanned by the collective variable $q$ ). The quality of this upper bound to the VAP energy depends on the relevance of the collective space for dynamical pairing correlations.

In a next step, we consider a more general N-body wave function defined as a linear superposition of projected HFB states with an unknown weight function $f_{j}(q)$ :

$$
\left|\Psi_{j}\right\rangle=\int \mathrm{d} q f_{j}(q)\left|\Phi_{N_{0}}(q)\right\rangle
$$

In this definition, the label $j$ recalls that several states $\left|\Psi_{j}\right\rangle$ are obtained corresponding to the correlated ground state and to the collective excited states. Within the GCM method, the function $f_{j}$ is determined by a variation of the total energy $E_{j}$

$$
E_{j}=\frac{\left\langle\Psi_{j}|\hat{H}| \Psi_{j}\right\rangle}{\left\langle\Psi_{j} \mid \Psi_{j}\right\rangle}
$$

with respect to the function $f_{j}^{*}(q)$. This leads to the HillWheeler equations [26]. When particle number projection is imposed on each basis state, the kernels $\mathcal{I}, \mathcal{N}$ and $\mathcal{H}$ of the integral operators entering this equation are given by

$$
\left(\begin{array}{c}
\mathcal{I}\left(q^{\prime}, q\right) \\
\mathcal{N}\left(q^{\prime}, q\right) \\
\mathcal{H}\left(q^{\prime}, q\right)
\end{array}\right)=\frac{1}{\pi} \int_{-\frac{\pi}{2}}^{\frac{\pi}{2}} \mathrm{~d} \phi \mathrm{e}^{-i \phi N_{0}}\left\langle\Phi\left(q^{\prime}\right)\left|\left(\begin{array}{c}
\hat{1} \\
\hat{N} \\
\hat{H}
\end{array}\right) \mathrm{e}^{i \phi \hat{N}}\right| \Phi(q)\right\rangle
$$

The evaluation of these $\left(q^{\prime}, q\right)$ dependent kernels is the most time consuming numerical part (in fact, there are four of them since neutron and proton numbers have to be conserved separately). They involve a double integral (one for $N$ and one for $Z$ ) of matrix elements of one-body and two-body operators between all possible states of the collective basis $\{|\Phi(q)\rangle\}$. The formulae useful for the calculation of the sets of matrix elements are given in Ref. [27]. Other kernels involving multipole moment operators associated with the various deformations are also needed for the computation of the shape properties of the collective GCM states. The reference just quoted also recalls how the solution of the Hill-Wheeler equation can be reduced to the diagonalization of an hermitian matrix computed from the kernels $\mathcal{I}, \mathcal{N}$ and $\mathcal{H}$.

The functions $f_{j}$ 's do not form an orthogonal set. Using the overlap kernel, one can define the functions $g_{j}$ 's

$$
g_{j}(q)=\left(\mathcal{I}^{1 / 2} \cdot f_{j}\right)(q)
$$

which are orthonormal and can be interpreted as collective functions in the usual sense. In Eq. (8), the notation
$\mathcal{I}^{1 / 2}$ stands for the integral operator whose square is equal to the integral operator with kernel $\mathcal{I}\left(q^{\prime}, q\right)$. In the next section, we present some functions $g_{j}$ associated with the quadrupole and octupole vibrations.

In the case of quadrupole vibrations, the GCM is solved separately in each representation of the permutation group of the three intrinsic axes. As discussed in Ref. [28, 29], the two independent representations of this group have different angular-momentum-parity $\left(J^{\pi}\right)$ contents: in the fully symmetric one, the major component of the collective wave function corresponds to $0^{+}$spin and parity, while, in the two dimensional representation, the collective N-body wave function is predominantly of the $2^{+}$type. Very recently, we have contructed an exact angular momentum projection code [30] and we have quantitatively checked the quality of this approximate spin projection. We have found that it is accurate whenever the extension of the collective function $g$ does not extend beyond the value $\left\langle Q_{20}\right\rangle=4 \mathrm{~b}$ of the quadrupole deformation. Since the influence of the spherical magic proton number $Z=82$, insures that $g$ is non zero only in the close vicinity of the $\left\langle Q_{20}\right\rangle=0$ point, this is the case for the $g$ functions of the lead isotopes.

Although our HFB and GCM codes allow triaxial deformations, the results presented below are based on collective bases including only prolate and oblate states. This is justified by the small extension of the collective wave functions. Nonetheless, all the GCM calculations presented below mix states located on the six semi-axes corresponding to the triaxiality angles $\gamma=0, \pm 60^{\circ}, \pm 120^{\circ}$ and $180^{\circ}$. We have tested the quality of this approximation by a performing full triaxial calculations on a few cases and we have checked that our conclusions do not depend on this degree of freedom.

The mean-field (and Lipkin-Nogami) wave functions spanning the collective spaces are constructed from an energy density functional whose particle-hole and particleparticle components are defined by means of two distinct effective density dependent two-body forces. For the sake of simplicity, we have presented the standard GCM formalism with a single effective Hamiltonian. In practice, we have adapted the GCM to the choice made in Sect. 2.1 and defined a generalized energy density to be used in the computation of the energy kernel $\mathcal{H}\left(q^{\prime}, q\right)$ given by Eq. (7). This involves both a prescription for handling the density dependence of the force and an extension of the energy density formalism to the non-diagonal matrix elements which appear in the integral in Eq. (7). We use the method described in Ref. [28] (for an alternative choice, see Ref. [31] for instance). The formulae for the two terms of the extended energy density are given in Ref. [28] and the implications and potential problems associated with this formulation are discussed in Refs. [32,24].

\section{Results}

We consider successively, the influence of correlations on the ground state properties and the collective excitations of five lead isotopes. 


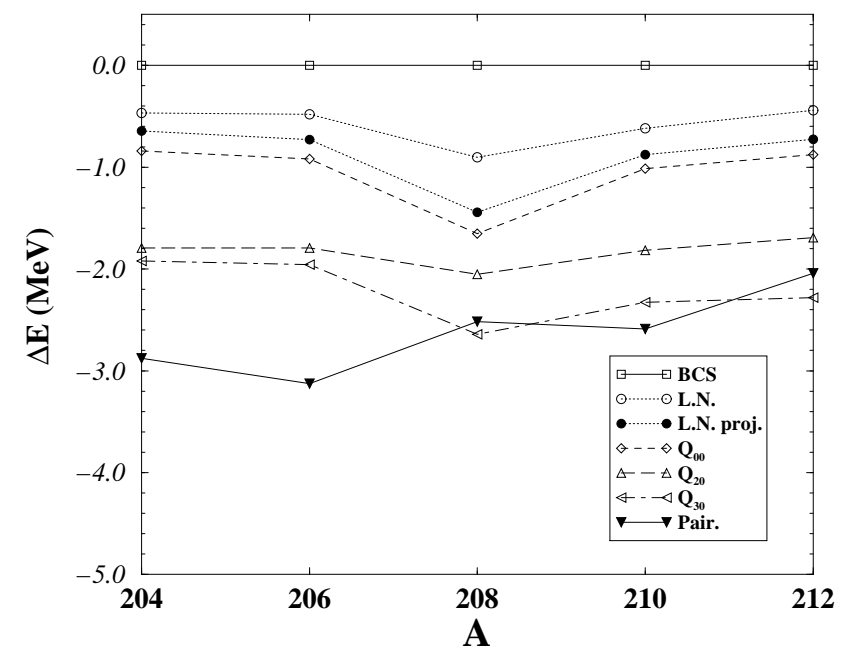

Fig. 1. Differences between the calculated total binding energy of the ground state of the five lead isotopes and a BCS reference. The labels $Q_{i 0}, i=0,2,3$ refer to GCM calculations taking into account the different types of shape collective excitations reported in Table1, while "Pair." stands for the pairing vibrations. Except for the curves BCS and L.N., all results involve an exact projection on $N$ and $Z$.

\subsection{Ground State Correlation Energies}

In Fig. 1, for each of the isotopes ${ }^{204} \mathrm{~Pb},{ }^{206} \mathrm{~Pb},{ }^{208} \mathrm{~Pb}$, ${ }^{210} \mathrm{~Pb}$ and ${ }^{212} \mathrm{~Pb}$, we have plotted the difference between the binding energies of the ground state calculated by GCM on several collective spaces and a BCS reference value. In practice, for the five nuclei, the proton part of this reference wave function reduces to a Slater (HF) determinant. This is also true for the neutron component in ${ }^{208} \mathrm{~Pb}$. In addition, in Fig. 1, we have plotted the difference between the same reference and the binding energies calculated by means of the Lipkin Nogami method with and without a projection on the neutron and proton numbers.

The ordinate scale of the figure shows that, irrespective of the nature of the collective vibration, the correction to the total binding energy is small (about $3 \mathrm{MeV}$ ). Considering the effective character of the two-body interactions defining the energy density, this is a satisfactory result. As we discussed in the introduction, one can consider either that, to first order effects of correlations can be effectively included into the parametrization of the force, or conversely that the presently available forces have to be readjusted so as to underbind slightly with respect to data. This would leave room for the small corrections associated with correlations to be computed on top of the mean-field solution, as we have done. In Ref. [33], much larger values of correlation energies have been found. However, as was pointed out in this reference, the method based on a sum rule approximation to the RPA suffers from an ambiguity related to the double counting in RPA formulas for the correlation energies. The GCM method is free from such a problem.
The relative magnitudes of the correlations tell about the nuclear sensitivity to various collective modes. For lead isotopes the octupole mode is the most efficient among the three nuclear shape vibrations analyzed in this work. One also notes that even for nuclei close to $N$ and $Z$ magic shell closures, pairing vibrations generate the largest correction.

The L.N. and even more the the L.N. projected curves show a larger effect for the doubly magic nucleus ${ }^{208} \mathrm{~Pb}$. For the latter curve, the difference with BCS increases from about $0.65 \mathrm{MeV}$ at ${ }^{204} \mathrm{~Pb}$ and ${ }^{212} \mathrm{~Pb}$ to $1.4 \mathrm{MeV}$ at ${ }^{208} \mathrm{~Pb}$. A possible interpretation is that the LN prescription which is designed to correct for vanishing pairing fluctuations in the HF+BCS solution, is more effective in ${ }^{208} \mathrm{~Pb}$ when the single particle energy gap at the Fermi surface is larger. With respect to the second curve (L.N.), the monopole, and octupole vibrations introduce an additional lowering of the energy whith an almost $N$ independent value equal to $0.2 \mathrm{MeV}$ and $1.4 \mathrm{MeV}$ respectively. By contrast, the curve associated with pairing vibrations shows an overall decrease versus $N$ of the binding energy correlations : over five isotopes it is reduced by about 0.6 $\mathrm{MeV}$.

In Fig. 2, we show the same results when the standard LN energy is taken as a reference. This presentation illustrates the improvements brought about by an exact projection as compared to the approximate prescription introduced by Lipkin and Nogami.

When the LN prescription does not yield a good approximation of the binding energy of the particle projected VAP solution, one could hope it to be a practical method to construct a BCS state close to the VAP intrinsic state. Then, a projection of this BCS wave function should give

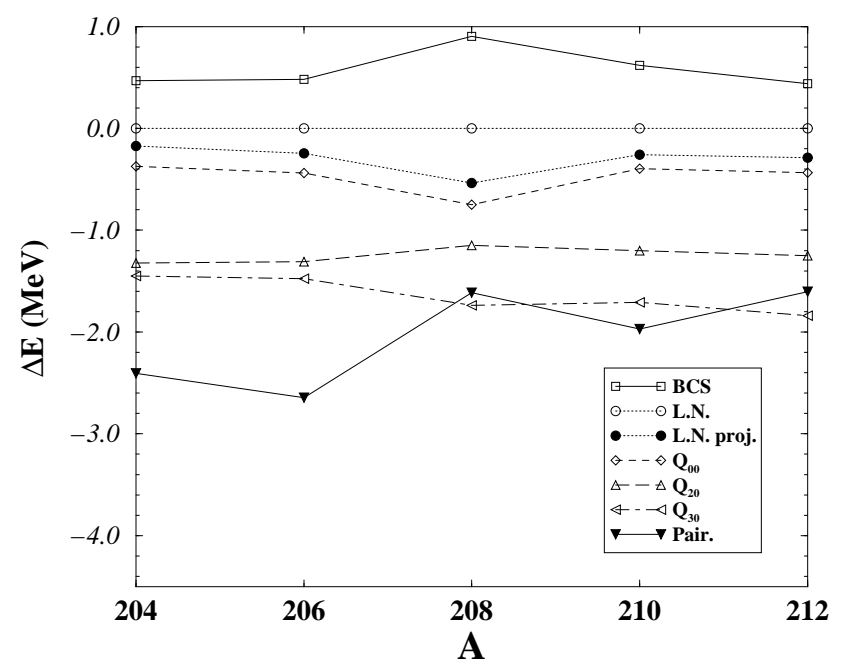

Fig. 2. Differences between the calculated total binding energy of the ground state of the five lead isotopes and the Lipkin Nogami reference. The labels $Q_{i 0}, i=0,2,3$ refer to GCM calculations taking into account various types of shape collective excitation as described in Table 1, while "Pair." stands for the pairing vibrations. Except for the curves BCS and L.N., all results involve an exact projection on $N$ and $Z$. 


\begin{tabular}{cccc}
\hline$A$ & $\begin{array}{c}\text { Lipkin-Nogami } \\
\left(\sqrt{\left\langle\Delta \hat{N}^{2}\right\rangle}, \sqrt{\left\langle\Delta \hat{Z}^{2}\right\rangle}\right)\end{array}$ & $\begin{array}{c}\text { Variat. Minimum } \\
\left(\sqrt{\left\langle\Delta \hat{N}^{2}\right\rangle}, \sqrt{\left\langle\Delta \hat{Z}^{2}\right\rangle}\right)\end{array}$ & $\begin{array}{c}\Delta E \\
(\mathrm{MeV})\end{array}$ \\
\hline 204 & $(2.54,1.14)$ & $(2.90,1.61)$ & -1.51 \\
206 & $(1.85,1.17)$ & $(2.60,1.95)$ & -1.36 \\
208 & $(1.36,1.17)$ & $(1.92,1.12)$ & -0.21 \\
210 & $(1.92,1.17)$ & $(2.37,1.13)$ & -0.84 \\
212 & $(2.59,1.17)$ & $(2.58,1.50)$ & -0.28 \\
\hline
\end{tabular}

Table 2. Neutron and Proton particle number fluctuations of the BCS states associated with the Lipkin Nogami solution and the minimum of the variational space constructed for the study of pairing vibrations (see text). The quantity $\Delta E$ give the difference between the energy of the latter state and the LN state after particle number projection has been effected (i.e. L.N. proj.).

almost the same energy as a VAP calculation. Our results show that the validity of this property of the LN prescription is at least nucleus dependent. The minimum of the energy surface obtained with the projected BCS states spanning the pairing collective space provides an upper bound of the VAP energy. From the results given in Table 2, it is seen that for the lighter lead isotopes, it is more than $1 \mathrm{MeV}$ below the energy associated with the projected Lipkin Nogami state. The same table shows that the lowest binding energies correspond to projected BCS states with particle number fluctuations larger than those predicted by the LN prescription. For ${ }^{208} \mathrm{~Pb}$ and isotopes above, the differences between the projected LN and approximate VAP solutions are smaller.

The discontinuity of the two-neutron separation energy $S_{2 n}$ at a shell closure predicted by mean-field methods is

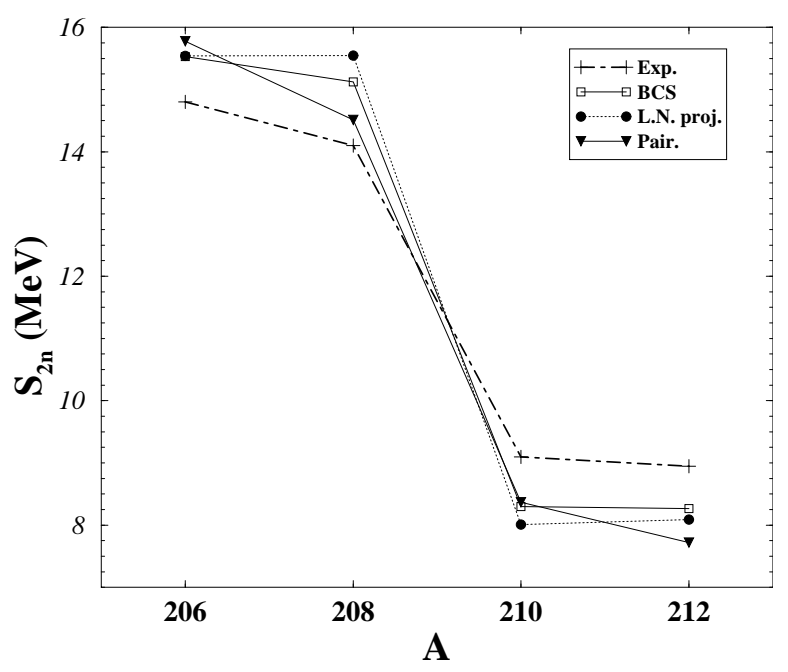

Fig. 3. Comparison between the experimental two neutron separation energies of the lead isotopes and the values calculated within the BCS, projected LN and GCM methods. In the latter case, the collective space allows fluctuations of the gap parameter.

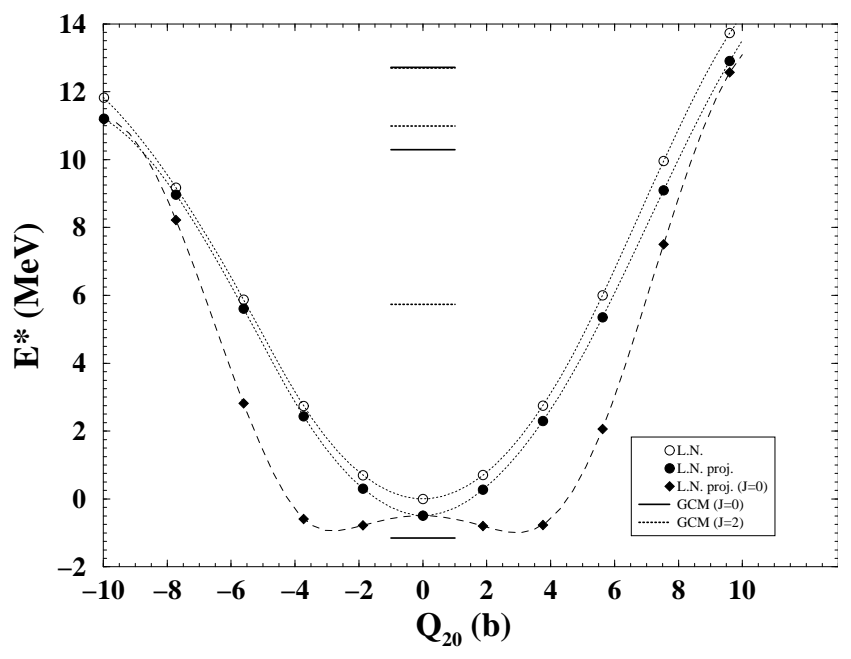

Fig. 4. Quadrupole deformation energy curves of ${ }^{208} \mathrm{~Pb}$ calculated with the Lipkin-Nogami state without or with exact projection on the neutron and proton particle number. In the latter case, the curve in the subspace containing the $0^{+}$component of the deformed function is also given. The solid straight segments give the GCM collective energy in this latter subspace, while the dotted segments give the energy in the subspace containing the $2^{+}$components of GCM the collective functions.

often larger than found experimetally. This effect can be seen in Fig. 3. The magnitude of the drop of the BCS $S_{2 n}$ value between ${ }^{208} \mathrm{~Pb}$ and ${ }^{210} \mathrm{~Pb}$ exceeds data by approximately $1.8 \mathrm{MeV}$. Although not shown in the figure, we have checked that the differences between the BCS and experimental $S_{2 n}$ 's are not larger than $0.2 \mathrm{MeV}$ for mass number $A$ smaller than 204. Therefore the larger discrepancy at $A=208$ cannot be ascribed to the asymmetry of the effective force SLy4 and suggests a deficiency of the $\mathrm{HF}+\mathrm{BCS}$ method for magic or near magic nuclei. This assumption is confirmed by the fact that the $S_{2 n}$ 's calculated with the GCM on a collective space allowing the fluctuations of the pairing gap leads to an improvement by about $0.6 \mathrm{MeV}$ (i.e. $33 \%$ ). By contrast, the Lipkin-Nogami prescription does not improve the mean-field results although it is supposed to approximately take into account the effect of these pairing vibrations. We have not plotted the curves with the energies resulting from the GCM associated with shape vibrations. Indeed, they are not much different from the LN curve.

\subsection{Collective Functions and Excitation Spectrum}

The $Z=82$ and $N=126$ magic numbers have a definite influence on the individual and collective properties of the five isotopes investigated in this work. They strongly favor a mean-field solution corresponding to a HF (no pairing) spherical wave function. The Lipkin Nogami prescription which transforms the HF states into $\mathrm{HF}+\mathrm{BCS}$ ones by enforcing a smooth decrease of the occupation probabilities from the hole to the particle states does not modify 
substantially the deformation properties of the collective energy surfaces. As it can be seen in Fig. 4, the LN energy curves of ${ }^{208} \mathrm{~Pb}$, with and without exact particle number projection, exhibit a well marked minimum at the spherical point. The curves are very similar, the main effect of the projection being a downward shift by about $0.5 \mathrm{MeV}$. When an approximate angular projection on the $0^{+}$state is performed, the bottom of the well is flattened over an extension of $\pm 4 \mathrm{~b}$. Qualitatively, such an effect is expected on general grounds. The LN wave function at the spherical point is a pure $0^{+}$state and is unaffected by projection while a deformed mean-field wave function is a mixture of different spin components. The energy of the $0^{+}$component is lower than that of all other spins and therefore than that of the deformed $\mathrm{HF}+\mathrm{BCS}$ wave function.

The GCM energies of the lowest collective quadrupole (approximate) $0^{+}$and $2^{+}$states of ${ }^{208} \mathrm{~Pb}$ are also plotted in Fig. 1. The first excited state is a $2^{+}$. A comparison with the GCM monopole calculation shows that the lowest $0^{+}$collective state in the spectrum of ${ }^{208} \mathrm{~Pb}$ is the one shown in Fig. 4, the energy of the first excited monopole GCM state being at $13.4 \mathrm{MeV}$. This latter value which does not vary with $N$ over the five isotope series, agrees with monopole giant resonance data 34, 35. This nice result is related to the nuclear matter incompressibility of the SLy4 force, $220 \mathrm{MeV}$, a value which is known to be coherent with experiment 36,37,38.

Fig. 5 displays the GCM probabilities (i.e. $|g|^{2}$ where $g(q)$ is defined by Eq. (8)) for the first $0^{+}$and $2^{+}$states. The former is peaked at the spherical point. Its width $(\approx 4 \mathrm{~b})$ corresponds to the quadrupole extension of the bottom of the angular momentum projected energy curve shown in Fig. A. The collective probability distribution of the first $2^{+} \mathrm{GCM}$ state displays a node at $Q=0$. Its

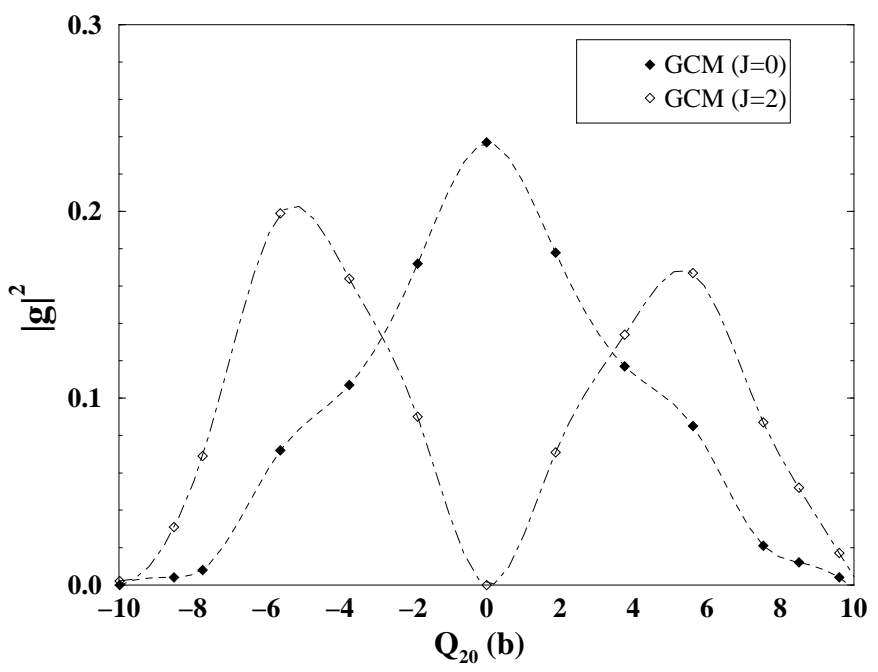

Fig. 5. GCM collective functions associated with quadrupole energy deformation of ${ }^{208} \mathrm{~Pb}$. The solid curve gives the wave function of the lowest energy state in the subspace containing the $0^{+}$component of the total wave function, while the dashed curve gives the same information for the subspace with the $2^{+}$ components.

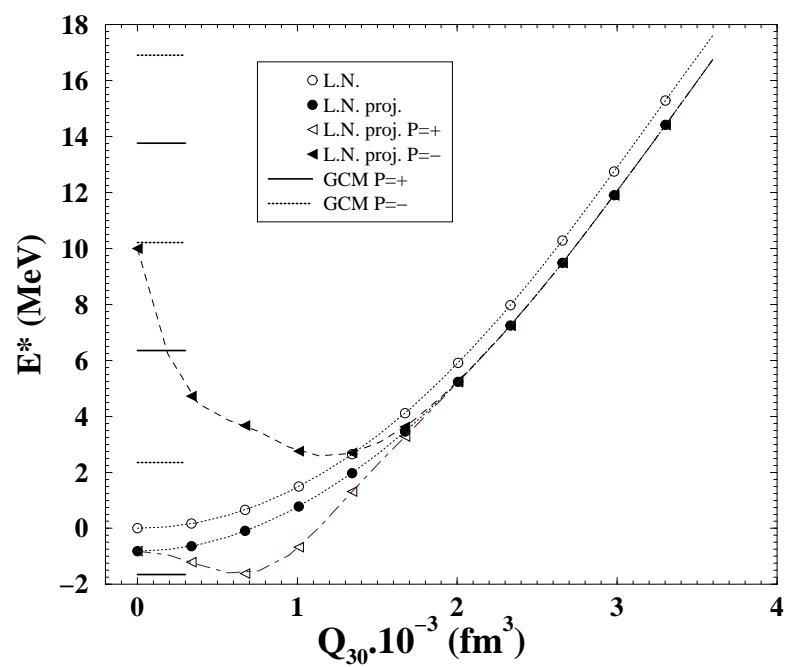

Fig. 6. Octupole energy deformation energy curves calculated with the Lipkin-Nogami state, without or with exact projection on the particle number. In the latter case, the curve in the subspace containing the positive and negative parity components of the deformed function is also given. The solid (resp. dotted) segments give the GCM collective energy in the positive (resp. negative) parity subspace of the GCM collective functions.

extension is almost symmetric on the prolate and oblate sides with a slight preference for the oblate deformation.

Four octupole deformation related energy curves for ${ }^{208} \mathrm{~Pb}$ are plotted in Fig. 6 against positive expectation values of the constraining operator $Q_{30}$ (see Table 1 ). Since this operator is parity negative, the negative abscissae part of each curve can be deduced by symmetry with respect to the ordinate axis. The LN and LN proj. curves display a minimum at the spherical point $\left\langle Q_{30}\right\rangle=0$. The projection merely increases the binding energy by $0.6 \mathrm{MeV}$. An additional projection of the wave function on the total positive (resp. negative) parity separates the subspace which contains the $0^{+}$(resp. the $3^{-}$) component of the LN particle projected state. The LN and LN proj. curves only differ significantly for small values of $\left\langle Q_{30}\right\rangle\left(<2.10^{3} \mathrm{fm}^{3}\right)$, namely as long as the overlap between the LN particle-projected wave function and its parity reversed is non zero. The positive parity curve has a minimum for $\left\langle Q_{30}\right\rangle \approx 10^{3} \mathrm{fm}^{3}$. Below, we will see that this number provides a measure of the octupole fluctuations in the $0^{+} \mathrm{GCM}$ state. We recall that symmetry garantees that the average value of $\left\langle Q_{30}\right\rangle$ vanishes exactly for all GCM states and, in addition, that the negative parity projected energy curve diverges at $\left\langle Q_{30}\right\rangle=0$.

The GCM energies given in Fig. 6 display an alternating sequence of positive and negative parity states. The first octupole GCM excited state should correspond to the lowest experimental $3^{-}$. Its probability distribution $\left(|g(q)|^{2}\right)$ and that of the $0^{+}$are plotted in Fig. 7. They give an information on the magnitude of the fluctuations in the corresponding collective state. Returning to Fig. 6, one checks that each of these two wave functions displays 


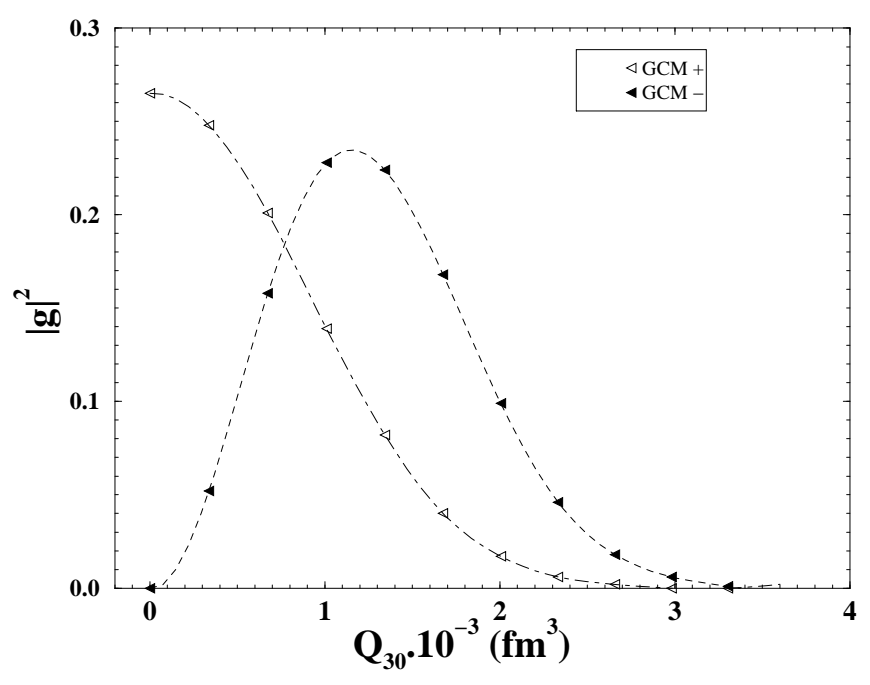

Fig. 7. Collective wave function of the lowest energy state with a given parity associated with the octupole deformation of ${ }^{208} \mathrm{~Pb}$. The solid (resp. dashed) curve gives the ground state function in the subspace containing the positive (resp. negative) parity component of the wave function.

the features expected for the quantum ground state in the projected Lipkin Nogami curve with the associated parity.

Up to now, the discussion in this subsection which centered on deformation energy curves and collective probability distributions has been concerned only with the doubly magic nucleus ${ }^{208} \mathrm{~Pb}$. The same curves calculated for the four other isotopes are qualitatively similar while, they yield significantly different collective energy spectra.Although one can discern evolutions of the energy curve versus $N$ which are at the origin of the differences found in the GCM spectra, they turn out to be neither spectacular nor linked to any specific part of the collective space. For this reason, we chose not to plot them; they only display a smooth modification of the same global pattern along the isotope series.

In Fig. 8, the collective GCM spectra for the three first excited states $\left(0^{+}\right.$and $2^{+}$quadrupole, $3^{-}$octupole) are given. Qualitatively, the overall pattern of data is well reproduced. The positive parity levels are pushed to high energy as one crosses $N=126$. As a consequence, the lowest collective state in ${ }^{208} \mathrm{~Pb}$ is a $3^{-}$. As one moves away from the shell closure, the collective spectrum returns to the usual midshell pattern with the $2^{+}$state being the lowest. However, the collective states whether quadrupole $\left(0^{+}\right.$and $\left.2^{+}\right)$or octupole are found by the GCM at energies between 1.5 to 2 larger than observed. Although the formalism of the GCM does not distinguish potential from collective inertia effects, if we were to adopt the concepts of the standard collective model to analyze our discrepancy with data, we could say that the results are consistent with the calculated moments of inertia of the low energy shape vibrations being twice too small. In the next section, based on the experience derived from Refs. [39, 40] and from the existence of significant dynamical pairing ef-

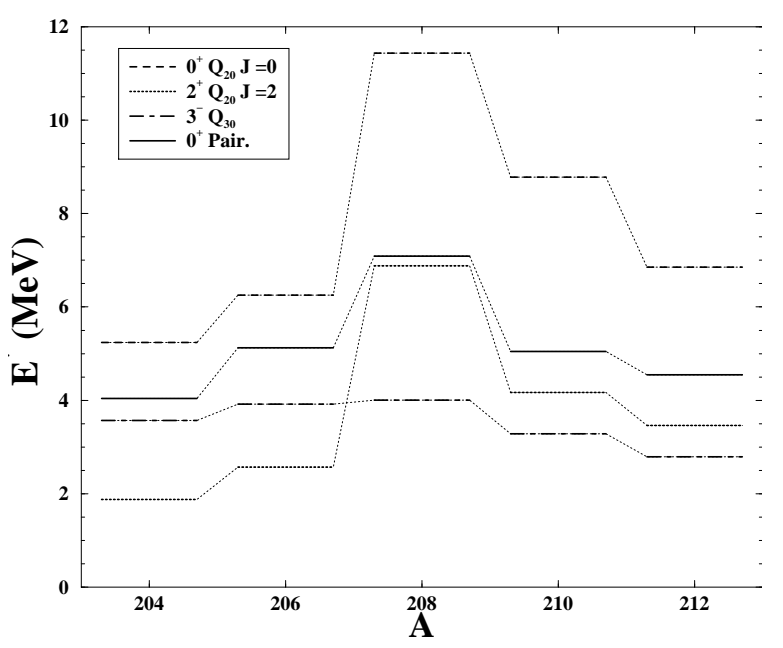

Fig. 8. Evolution of the lowest excited collective states of five lead isotopes near the magic nucles ${ }^{208} \mathrm{~Pb}$. The figure compares data with results of GCM including an exact projection on particle $(N$ and $Z$ ) numbers and an aproximate projection on angular momentum.

fects evidenced by the results discussed in Sect. 3.1, we put forward possible explanation for the GCM results.

With the Skyrme interaction SLy4, the quadrupole collective transition matrix element ${ }^{208} \mathrm{~Pb} B\left(E_{2}\right) \uparrow=7.1 \mathrm{~W} . u$. is close to the data ( $8 \mathrm{~W} . \mathrm{u}$.). The agreement is as good for the octupole transition since we find $B\left(E_{3}\right) \uparrow=18.7 \mathrm{~W} . u$. as compared to the experimental value equal to $32 \mathrm{~W} . u$. [34, 41, 42, 35]. We note however that these results are very sensitive to the interaction since a similar calculation with the SGII interaction yields 14 W.u. and 58 W.u. respectively.

\section{Conclusions and Perspectives}

We have performed an analysis of the collective correlations and modes of excitations in the vicinity of the magic nucleus ${ }^{208} \mathrm{~Pb}$. One of the characteristics of this work is an attempt to maximize the coherence of the description of the mean-field and collective effects for any amplitude. This is done by means of the GCM solved in collective bases. Depending on the type of collectivity, the GCM bases are obtained from constrained calculations (shape vibration) or derived from a set of auxiliary Hamiltonians (pairing vibrations). Another element of consistency is the use of the same effective particle-hole $(p-h)$ and particleparticle ( $\mathrm{p}-\mathrm{p})$ interactions in the complete sequence of calculations. These two forces had been determined in earlier analyses of ground state and high spin properties by means of mean-field methods.

We find that correlation effects on the total binding energies are small. A posteriori, this result justifies the perturbative-like calculation of such correlations on top of the mean-field solution as done in the present work. It also supports attempts at the definition of a force to be 
used exclusively in mean-field calculations and designed to effectively incorporate the small effect of correlations [10,11. The latter type of force is a practical tool for the extensive nuclear mass tabulations required for a global analysis of astrophysical nucleosynthesis.

Furthermore, we have found that pairing vibrations induce a softening of the rapid variation of the $S_{2 N}$ curves at the crossing of the magic shell $N=126$. Such accidents of the nuclear mass chart have a significant influence on the understanding of nuclear abundance peaks resulting from high neutron fluxes in stellar events such as class II supernovae explosions. A check of the persistence of this effect at the points where the r-process path crosses the $Z=82$ magic line (on the $N$-rich side) appears therefore desirable.

Our work also provides an illustration of the validity of the Lipkin Nogami prescription. The LN method should give good approximations of both the VAP wave function and energy. More restrictively, it is considered that even when the LN energy does not reproduce well the VAP energy, an exact projection performed on the LN wave function does so 43. This implies that VAP is well approximated by a projection after the solution of the LN equations. Our calculations show the limitations of these two assumptions. We find that even after projection the discrepancy on the binding energy is nucleus dependent and can be larger than $1.0 \mathrm{MeV}$. Our calculation provides only a lower bound of this discrepancy since the variational space used in the present work only covers a fraction of the space available to a full VAP calculation

We find that, for the five lead isotopes, the GCM collective vibrations associated with our variational spaces only generate small fluctuations around the spherical shape and do not modify significantly the expectation values of multipole moments. The behaviour of the low energy collective spectrum is qualitatively correct. In particular the relative positions of the $0^{+}, 2^{+}$and $3^{-}$states along the isotope sequence is well reproduced. We also find a reasonable agreement on electromagnetic transition matrix elements. However, the energy scale of our spectrum is too large by a factor between 1.5 and 2 compared to data. The reason for this discrepancy is not yet clear. This is all the more disconcerting that RPA calculations on the Hartree-Fock solution using the same interaction provide a much better agreement with the low energy collective spectrum of ${ }^{208} \mathrm{~Pb}$ 44. For this nucleus, we have additionally performed a GCM calculation of quadrupole and octupole vibrations using the SGII interaction for which RPA calculations are also available [45]. We find a similar pattern of fair agreement on transition matrix elements and discrepancy on collective spectrum energies.

From these results, some directions for further work can be outlined. First it appears useful to extend the calculation to other regions of the mass table to test the importance of pairing vibrations at shell closure. In particular one should consider other proton magic numbers with a focus on the neutron rich side at places of interest for the r-process. Second, one sould extend the formalism with a coupling between the vibrational modes which have been detected to be especially active. For the lead isotopes, the first such investigation should be devoted to a coupled calculation of pairing and octupole vibrations.

An other point which needs clarification is the discrepancy on the low energy collective spectrum density. The problem does not seem related with the interaction since RPA provides a rather good agreement with data. We note also that with the same forces, GCM and RPA results on giant resonances agree together (and also with data). A first reason for the differences on low energy collectivity may be due to the use of a GCM basis of wave functions including dynamical pairing while RPA is performed on a Hartree Fock solution.

A discussion of this problem can be found in [39,40]. Indeed, in cranking type calculations, pairing reduces collective mass parameters and leads to a decompression of the spectrum. In such a case, one would have to reconsider which fraction of the pairing must be assigned to static (i.e. BCS or HFB) or dynamic (fluctuations of the magnitude of pairing gap) correlations. Another source for the different GCM and RPA results may be due to the definition of the GCM collective spaces. To study shape vibrations, we have used a collective basis generated by self consistent calculations with constraining operators proportional to multipole moments. A description of the low energy mode could require other radial form factors more peaked at the nuclear surface than the present $r^{n}$ form factors. The RPA transition form factors may provide indications for more appropriate constraining operators.

As a conclusion, the present work has demonstrated the interest of our approach and also shown some of its limitations in its present form. Nevertheless we believe that it establishes that the GCM performed on many-body bases including exact symmetries such as particle number, parity (as is done here) but also angular momentum has strong potential for the description of nuclear properties which remains largely to be explored.

\section{Acknowledgements}

We thank P.-G. Reinhard and G. L. Colo for providing us information on specific RPA calculations. This work has been supported in part by the PAI-P3-043 of the Belgian Office for Scientific Policy.

\section{References}

1. P. Bonche, H. Flocard, P.-H. Heenen, S. J. Krieger, M. S. Weiss, Nucl. Phys. A443, 39 (1985).

2. P. Fallon, P.-H. Heenen, W. Satuła, R. M. Clark, F. S. Stephens, M. A. Deleplanque, R. M. Diamond, I. Y. Lee, A. O. Macchiavelli, K. Vetter, Phys. Rev. C 60, 044301 (1999).

3. D. Gogny, Nuclear Self Consistent Fields, ed. G. Ripka, M. Porneuf, (Amsterdam, North Holland, 1975) page 333.

4. J. Dechargé, D. Gogny, Phys Rev. C 21, 1568 (1980).

5. S. Péru, M. Girod, J. F. Berger, Eur. Phys. J. A. 9, 35 (2000). 
6. S. A. Fayans, S. V. Tolokonnikov, E. L. Trykov, D. Zawischa, Nucl. Phys. A 676, 49 (2000).

7. P. Ring, Prog Nucl. Part. Phys.37, 193 (1996).

8. G. A. Lalazissis, J. König, P. Ring, Phys. Rev. C 55, 540 (1997).

9. V. Bernard, Nguyen Van Giai, Nucl. Phys. A 348, 75 (1980).

10. F. Tondeur, S. Goriely, J. Pearson, M. Onsi, Phys. Rev. C 62, 024308 (2000).

11. A. Brown, Phys. Rev. C 58, 220 (1998).

12. S. J. Krieger, P. Bonche, H. Flocard, P.-H. Heenen, R. Mehrem, M. S. Weiss, Phys. Rev. C 54, 2399 (1996).

13. J. Skalski, P.-H. Heenen, P. Bonche, H. Flocard, J. Meyer, Nucl. Phys. A551, 109 (1993).

14. P.-H. Heenen, J. Skalski, Phys. Lett. B381, 12 (1996).

15. H. J. Lipkin, Ann. Phys. (N.Y.) 9, 272 (1960).

16. Y. Nogami, I. J. Zucker, Nucl. Phys. 60, 203 (1964).

17. H. C. Pradhan, Y. Nogami, J. Law, Nucl. Phys. A201, 357 (1973).

18. J. Dobaczewski, H. Flocard, J. Treiner, Nucl. Phys. A422, 103 (1984).

19. E. Chabanat, P. Bonche, P. Haensel, J. Meyer, R. Schaeffer, Nuc. Phys. A635, 231 (1998).

20. C. Rigollet, P. Bonche, P.-H. Heenen, H. Flocard, Phys. Rev. C 59, 3120 (1999).

21. S. Ćwiok, W. Nazarewicz, P.-H. Heenen, Phys. Rev. Lett 83, 1108 (1999).

22. J. Terasaki, P.-H. Heenen, P. Bonche, J. Dobaczewski, H. Flocard, Nuc. Phys. A593, 1 (1995).

23. T. Duguet, P. Bonche, P.-H. Heenen Nucl. Phys. A679, 427 (2001).

24. L. Egido, private communication.

25. J. Meyer, P. Bonche, J.Dobaczewski, H. Flocard, P.-H. Heenen, Nucl. Phys. A533, 307 (1991).

26. D. L. Hill, J. A. Wheeler, Phys. Rev. 89, 1102 (1953).

27. P. Bonche, J. Dobaczewski, H. Flocard, P.-H. Heenen, J. Meyer, Nucl. Phys. A510, 466 (1990).

28. P. Bonche, J. Dobaczewski, H. Flocard, P.-H. Heenen, Nucl. Phys. A530, 149 (1991).

29. P.-H. Heenen, P. Bonche, J. Dobaczewski, H. Flocard, Nucl. Phys. A561, 367 (1993).

30. A. Valor, P.-H. Heenen, P. Bonche, Nucl. Phys. A671, 145 (2000).

31. A. Valor, J. L. Egido, L. M. Robledo, Phys. Lett. B392, 249 (1997).

32. N. Tajima, H. Flocard, P. Bonche, J.Dobaczewski, P.-H. Heenen, Nuc. Phys. A542, 355 (1992).

33. P.-G. Reinhard, J. Friedrich, Z. Phys. A321, 619 (1985).

34. S. Brandenburg et al., Nucl. Phys. A466, 29 (1987).

35. D. H. Youngblood, H. L. Clark, Y. W. Lui, Phys. Rev. Lett. 82, 691 (1999).

36. J. P. Blaizot, Phys. Repts 64, 171 (1980).

37. J. Speth, Electric and Magnetic Giant Resonances, (World Scientific, Singapore, New Jersey, London, Hongkong, 1991).

38. J. P. Blaizot, J. F. Berger, J. Dechargé, M. Girod, Nucl. Phys. A591, 435 (1995).

39. L. Próchniak, K. Zając, K. Pomorski, S. G. Rohoziński, J. Srebny, Nucl. Phys. A648, 181 (1999).

40. K. Zajạc, L. Próchniak, K. Pomorski, S. G. Rohoziński, J. Srebny, Nucl. Phys. A653, 71 (1999).

41. C. Djalali et al., Nucl.Phys. A380, 42 (1980).

42. M. J. Martin, Nuclear Data Sheets, 47, 850 (1986).
43. P. Magierski, S. Ćwiok, J. Dobaczewski, W. Nazarewicz, Phys. Rev. C 93, 1686 (1993).

44. P.-G. Reinhard, private communication.

45. G. L. Colo, private communication. 